\title{
Management of an ATM based Integrated Voice and Data Network - a Pragmatic Solution
}

\author{
Werner Filip \\ IBM European Networking \\ Center \\ Vangerowstr. 18 \\ D-69115 Heidelberg \\ Germany \\ teleph. +49-6221-59-4371 \\ fax +49-6221-59-3300 \\ wfilip@vnet.ibm.com
}

\author{
Georg Zoerntlein \\ IBM European Networking \\ Center \\ Vangerowstr. 18 \\ D-69115 Heidelberg \\ Germany \\ teleph. $+49-6221-59-4389$ \\ fax +49-6221-59-3300 \\ gzoern@vnet.ibm.com
}

\begin{abstract}
The realization of management systems for large, heterogeneous telecommunication networks is still an open issue with hard to tackle problems. This paper reports on the architecture, design and implementation of a management for a large scale ATM based integrated voice/data network. The focus has been put on the description of a pragmatic approach to implement a standardized, TMN-based solution. The approach is being called pragmatic since various existing standardized generic building blocks have been employed for the implementation. Additionally, the functionality of already existing element management systems for the various network components has been exploited and integrated in a homogeneous user environment. Thus, it was possible to save a lot of cumbersome implementation work.
\end{abstract}

\author{
Keywords \\ ATM, Voice and Data Network, TMN, VPN, OMNIPoint, CMIP
}

\section{INTRODUCTION}

New network providers and already established public network operators (PNOs) are starting to exploit advanced technologies, most prominently ATM, in a large scale. The goal is always to provide end-users with new types of services (e.g. video conferencing, enhanced voice services, corporate networks etc.), and to lower transmission costs by optimizing bandwidth utilization. A large scale exploitation of advanced network technology, means, at least currently, that it has to be integrated into an existing network infrastructure or combined with network components that implement an already well established network service like the conventional voice phone. Additionally, a powerful 
and extendible network management system is needed to fully bring to bear the new technology and to achieve the aspired goals.

The requirements on such a network management system are tremendous, comprising all hard to tackle issues of network management, as there are the most dominant ones:

- appropriate visualization of large networks,

- handling of huge data volumes describing the network,

- heterogenity of the network components with different management interfaces,

- diversity of the services to be managed, and

- complicated technical interrelationships of the various hardware components.

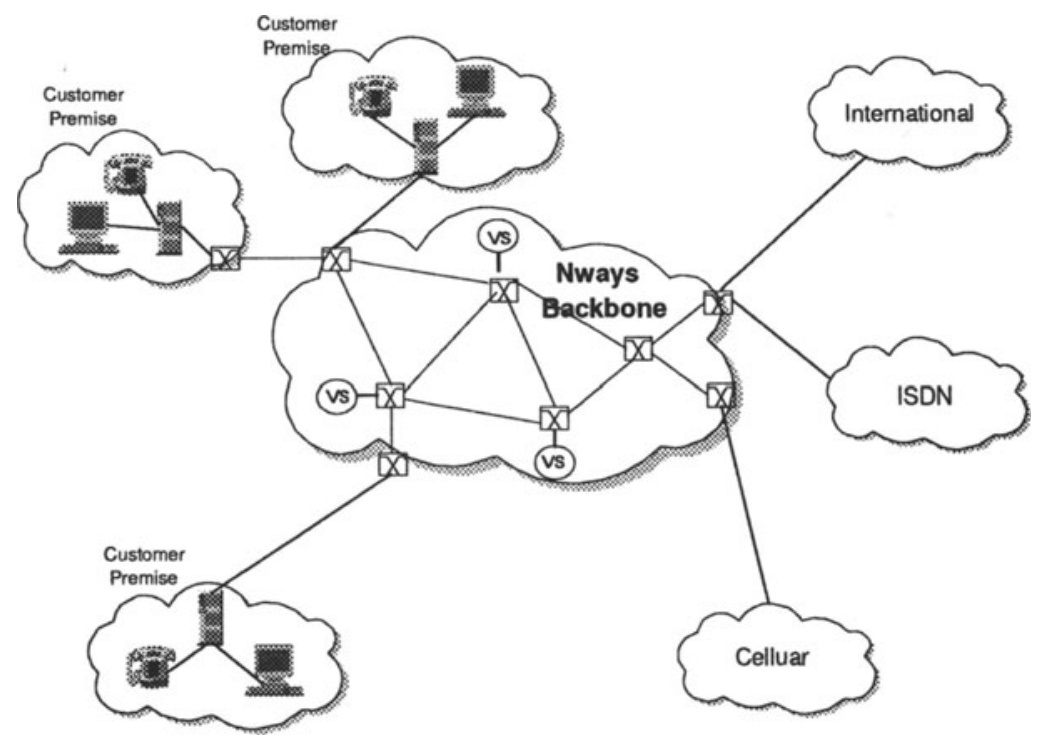

Figure 1 Integrated Voice/Data Network

In this paper the architecture, design and realization of the network management system for a large scale, integrated voice/data network will be described. Most notably, the focus will be on the description of the pragmatic approach that has been chosen to implement the network management system. The approach is being called pragmatic since various existing generic but standardized building blocks have been exploited for implementing the network management solution. This was the only way a solution for a network of the given complexity could be provided which was cost effective and implementable in a timely fashion.

To make our explanations and reasoning clear a short outline of the network that has to be managed and its components is given. This network is being implemented country-wide by an upcoming German public network provider (PNO).

In Figure 1 the main components of the integrated voice/data network and their composition is illustrated. The basis for transferring data and voice traffic is an ATM backbone network that consists of ATM switches which are connected via E1 and/or E3 trunks. The ATM switches provide various adapters establishing different data transfer interfaces (e.g. X.25, FrameRelay etc.). For providing voice services dedicated 
voice switches are employed. Those voice switches implement the appropriate means for handling phone calls, maintaing subscriber numbers and do signalling between voice switches.

For both types of switching elements in the network (ATM switches and voice switches) element management systems are available providing convenient end-user interfaces (EUI) to initiate management commands. It was an important goal in the design of the integrated management solution to exploit the existing element management systems appropriately to avoid the reimplementation of already existing management functionality.

The paper has been organized in the following way: In the next section an overview on the requirements of the integrated network management system (INMS) will be given. The third section summarizes the principal architectural concepts on which the implementation of the INMS has been based on. The way, the different management layers have been structured and implemented is presented in the fourth section. The functionality of the INMS applications is sketched in the fifth section. The final section summarizes our experiences and results.

\section{REQUIREMENTS ON INTEGRATED NETWORK MANAGEMENT}

It has been pointed out that for the two component types of the described voice/data network two element management systems already exist. Each management system allows to manage networks built up from a single component type (voice switches or ATM switches) in a convenient way. However, the management of a network that is composed of the two different network element types is not facilitated properly by either of the two management systems. Also, a disjoint use of the both management systems will not provide the proper means to manage a composed network especially a network of the size envisioned by the network provider. Thus, it has been decided to implement an integrated network management system (INM) providing an operator with a single view on the composed network and a uniform operator interface for both types of network elements.

Considering the envisioned size and complexity of the integrated voice and data network essential criteria for the integrated network management system (INMS) have been:

\section{Functionality}

- Integrated Fault Management - All fault messages initiated from the various equipment types should be represented in a standardized format and represented homogeneously. Thus, it will be possible to interface with various tools and fault management applications in a uniform way.

- Integrated Network Configuration Management - The INMS has to provide the functionality to configure network topologies composed of the different network element types. Via an appropriate graphical representation of the topology the invocation of functionality for network element configuration should be uniform and simple.

- Definition of Views - The INMS has to provide the capability to define dynamically different views (e.g. voice view, flat view etc.) which allow for different types of network operators to concentrate on specific parts of the network. Since the network will grow over time it has to be possible to adapt the network management organization dynamically.

- Customer Network Management Services - The INMS has to provide appropriate means that a customer can retrieve management information for his dedicated net- 
work parts (access nodes). Throughout this paper these services will also be synonymously denoted under the term VPN (virtual private network).

\section{Simple uniform interface}

Operators should not deal with different tools for different network management functions, because this leads to high education efforts, acceptance problems, and network management operations. A uniform interface will guarantee that operators have a high productivity and a steep learning curve.

\section{Scalable architecture}

The architecture must allow that the management system scales appropriately with the size of the network and the number of customers on the network.

\section{Extendability}

It must be possible

- to easily integrate functions for managing new resources,

- to introduce new customer services quickly, and

- to facilitate organizational changes appropriately.

\section{MANAGEMENT OF TELECOMMUNICATIONS NETWORKS}

The management and administration of large and complex networks, as the one outlined in the introduction, asks for a well architected system. The standards and architecture of choice for the world market is called the Telecommunications Management Network (TMN) as specified by the ITU-T standards body [1], [2], [7]. This standard provides both an operational and a technical model for network management in the telecommunications environment that is intended to allow for consistent network management of equipment and services regardless of the vendor. The operational model describes the following four layers from lowest to highest:

- Network Element Management - These network managers manage specific pieces of equipment such as switches, multiplexors, etc.

- Network Management - These managers combine the management of Network Element Managers to allow for the management of a specific network.

- Service Management - The Service Managers manage across networks to provide services to the customer, e.g., leased line and telephone. They also can provide a window for their customer into the service provider's company.

- Business Management - This is the administration layer of management and takes care of functions such as billing, receivables, etc.

The technical models are in the form of strictly defined connectivity both between the layers and within the layers and object representations or abstractions of what is being managed. This connectivity has been defined though very detailed profiles of international standard protocols that platform providers and equipment manufacturers will be required to implement.

The operational layers talk to each other in a hierarchy. For example, the Network Element Managers have manager applications that manage the Network Elements (e.g., switches). The software in the network element that corresponds with the management applications in the Network Element Manager is called an agent and, therefore, creates an agent manager relationship between the two layers.

The object models are abstractions of both physical (e.g., switches) and logical (e.g., services) entities that will be managed. These abstractions not only describe the attributes of the entity but the way the entity behaves, how it can be monitored, and 
how it can be controlled. These definitions are being described in a specification language (GDMO) [4] that can be utilized by application programs to implement both management and agent applications.

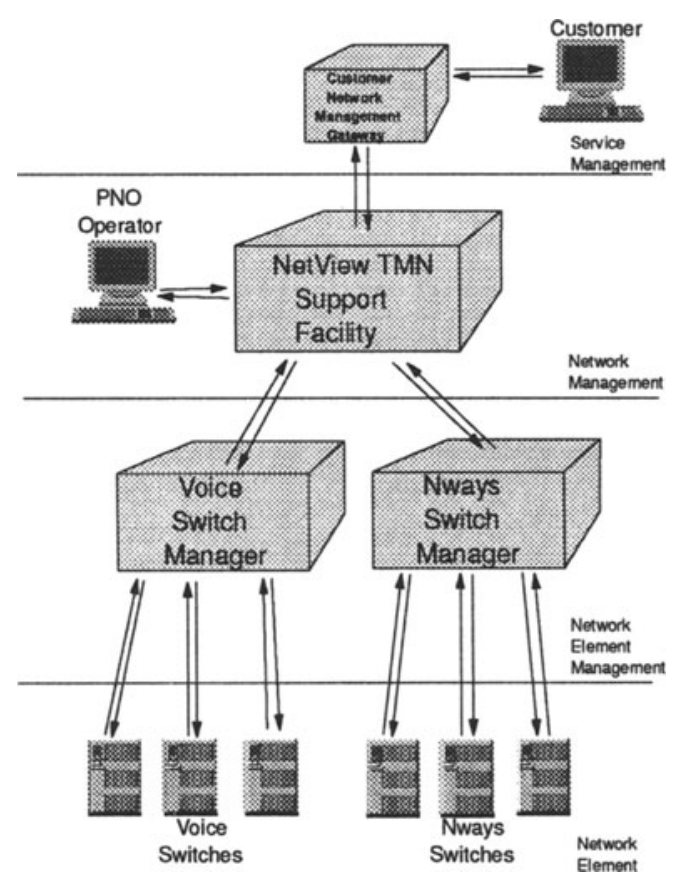

Figure 2 INM Architecture

Following the standards the architecture depicted in Figure 2 has been chosen as the basis for realizing the INMS. IBM's NetView TMN Support Facility for AIX (TMN/6000) [8], [9], [10] with its special extensions for managing telecommunications networks has been utilized to implement the functions of the different management layers. A detailed description of the various agents that have been implemented will be given in the following chapter.

\section{IMPLEMENTATION OF THE MANAGEMENT LAYERS}

\section{Agent Structure}

The implementation concept of the INMS is primarily based on the exploitation of the X.700 Agent/Manager concept. This means that a hierarchy of Agent/Manager components are realized which finally implements the user functionality that is described in the next chapter.

An Agent/Manager component can be characterized in the following way: It acts as a manager (i.e. sending CMIP requests) to the components which are at a lower level in a Agent/Manager hierarchy and behaves as an agent (i.e. answering to CMIP 
request and sending event reports) to higher layer Agent/Manager components. An Agent/Manager component contains objects which are instantiations of object classes encapsulating data attributes as well as behavioural actions.

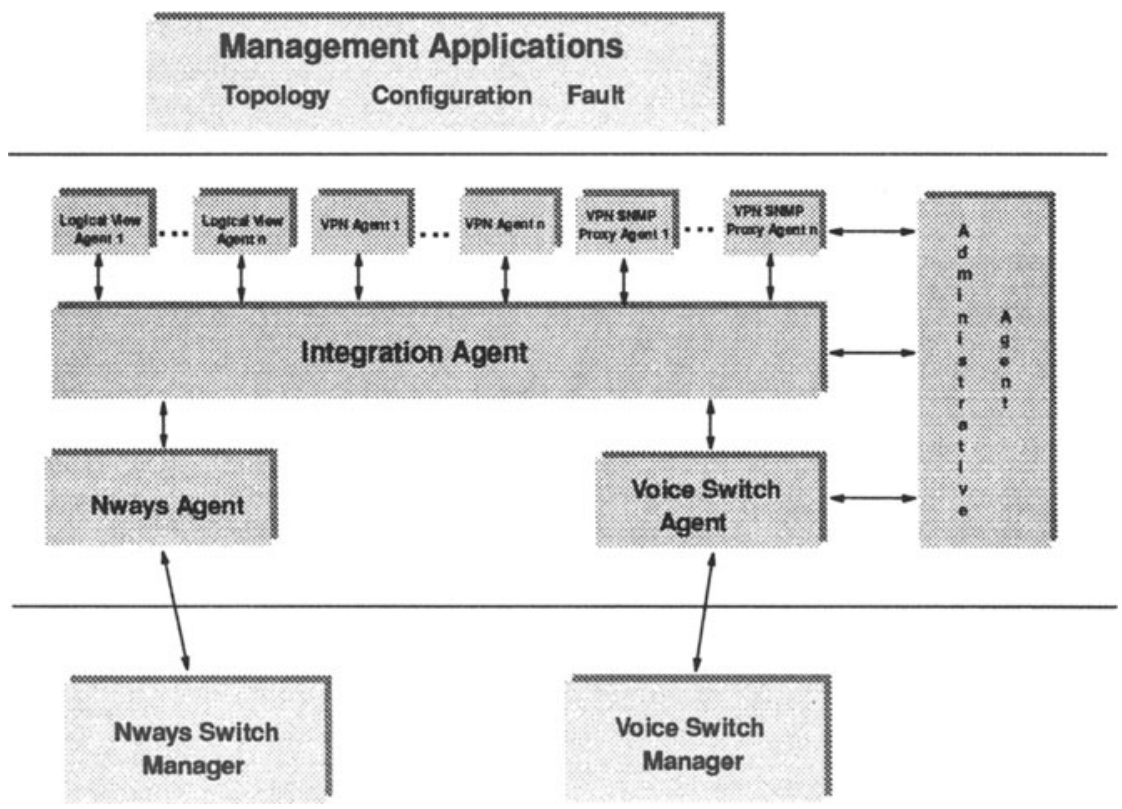

Figure 3 Agent/Manager Structure

The TMN platform provides an environment and a framework which supports the implementation of Agent/Manager components. Compiler tools are provided that transform a GDMO based managed object (MO) specification to a large degree into executable code that linked with an agent runtime environment executes the described functionality. A specific editor is available which allows to extend the automatically generated code appropriately. Thus, hand crafted code can be integrated conveniently for those implementation aspects that could not be derived automatically from the semiformal GDMO specifciation. The object classes can optionally be generated as persistent code interfacing to an object oriented database system. Based on this environment the Agent/Manager hierarchy depicted in Figure 3 has been implemented. The different Agent/Manager components realize the following functionality:

- Integration Agent - The Integration Agent contains objects that model the physical topology of the interconnected voice and ATM switching elements.

- Logical View Agent - Logical View Agents represent alternative logical views on top of the physical topology. This allows an operator to focus on specific network parts.

- Voice Switch Agent - The Voice Switch Agent realizes a kind of Q-adapter functionality for the voice switch network elements, maintaining all information to allow the integrated use of the element management functionality provided by the voice switch manager (VSM).

- Nways Agent - The Nways Agent realizes Q-adapter functionality for the Nways network elements and maintains all information to invoke the functionality of the Nways switch manager (NSM) 
- VPN Agent - The VPN Agents are special customer specific view agents which focus on the access nodes for a customer and their connections into the PNO network.

- VPN SNMP Proxy Agent - A VPN SNMP Proxy Agent provides an SNMP gateway for a VPN agent.

- Administrative Agent - The VPN Adminstrivative Agent contains all administrative information (addresses, entry objects etc.) for the various agents in the system.

\section{Object Definitions}

The objects establishing a topological view on the network have been derived from the following classes of the OMNIPoint Management Information Base (MIB) [5],[6]:

- representing links (objects that transport information)

- circuit - a connection between two nodes; it may consist of component circuits.

- facility - refers to the physical means carrying a signal; used to carry circuits.

- transport connection - established and used by two peer connection oriented transport protocol layer entities for transferring data.

- representing nodes (objects that generate/consume information)

- opEquipment - represents physical components of a managed element;

- computerSystem - represents the aggregate of components which as a whole is capable of performing data processing, storage, and retrieval functions.

- processingEntity - represents the physical portion of a computer system;

- coTransportProtocollayerEntity - represents an instantiation of any connection-oriented transport layer protocol.

- cITransportProtocolLayerEntity - represents an instantiation of any connectionless transport layer protocol.

- location - refers to a place occupied by one or more objects or persons;

- representing collections of links and nodes (supporting hierarchy levels)

- opNetwork - represents collections of interconnected telecommunications and management objects; an opNetwork may be nested within another opNetwork.

customer - refers to a corporation, organization or individual.

\section{INM MANAGEMENT APPLICATIONS}

In the following the functionality of the INM management applications will be characterized as they are available to the network operator. Those applications interact with the CMIP agents described in the previous chapter.

\section{Integrated Topology}

The INM Topology will act as an integrated graphical user interface for operators to perform various network management functionality. Different topologies can be implemented via view agents and used in parallel. The topology display is based on the OMNIPoint object model. The OMNIPoint object model has been chosen, because it already supports the object class "customer", which provides the basis for the introduction of Virtual Private Networks (VPNs). The GUI will

- display different locations of network devices represented by a location symbol;

- show connections between locations and phys ical network nodes displayed as lines;

- display the status of a symbol or a line using different colors (customization is possible).

- allow to explode location symbols (double clicking), displaying their respective functional components which can be access nodes, backbone nodes, or a combination of both. If there is more than one functional unit associated with a location, the connections between those functional units are also displayed. 
- show access and backbone nodes represented by different symbols (again color coding is used to indicate status information)

- provide the functionality to execute voice equipment and Nways switch symbols. That means, double-clicking on a symbol could start the appropriate management application for the real resource represented by the symbol (dependent on the element managers' supported functions). Thus, the topological views act as an integration point for the different tools managing the different systems in the network.

In the following a concrete topology as reflected in the GUI will be presented. The topology is implemented by a View Agent which is based on the Integration Agent (see Figure 4 for details).

\section{The PNO Icon}

An icon, representing the PNO enterprise, will be presented in the sub-map as a result of double-clicking on the OMNIPoint icon (view 0 ).

\section{Exploding the PNO Icon}

The operator can double-click on that icon and a sub-map, showing icons representing the major groups of information available through the PNO topology navigation tree, will be presented as shown in view 1 . These icons are the following:

- One icon representing an instance of the [PN]:pnAgents class which contains instances representing the various agents.

- One icon representing an instance of the [PN]:pnIntegratedNetwork class containing the PNO Integrated Network Hierarchy.

- One icon representing an instance of the [PN]:pnLogicalView class containing the hierarchies of existing Logical Views.

- One icon representing an instance of the [PN]:pnVpNetwork class containing the PNO customers and its VPN Views.

All these instances are contained in the Administrative Agent (see previous section for more details).

\section{Exploding the Integrated Network Icon}

When exploding this icon, a sub-map with icons representing major portions, sub-networks, of the PNO Integrated Network will be presented. Such icons represent instances of the [PN]:pnNetwork class. Any icon that represents such an instance of the [PN]:pnNetwork class can again be exploded into a sub-map showing the next level of details of the PNO Integrated Network as depicted in view 3 which shows a sub-map with the details of the 'FRANKFURT' network shown in view 2 . Such submap, with the details of a network as shown in views 2,3 and 4, can contain icons representing instances of the following classes:

- $[\mathrm{PN}]:$ pnNetwork

- [PN]:pnBackboneNode

- [PN]:pnAccessNode

\section{Exploding Links between Networks and Nodes}

As shown in views 2, 3 and 4, networks and nodes represented by such icons can be connected with a line representing the fact that at least one direct link exists connecting two equipments where one of these equipments belongs to the element represented by one of the icons and the other equipment belongs to the element represented by the other icon. Such lines, will always represent instances of the class [PN]:pnCircuit, contained in the PNO Integration Agent. 


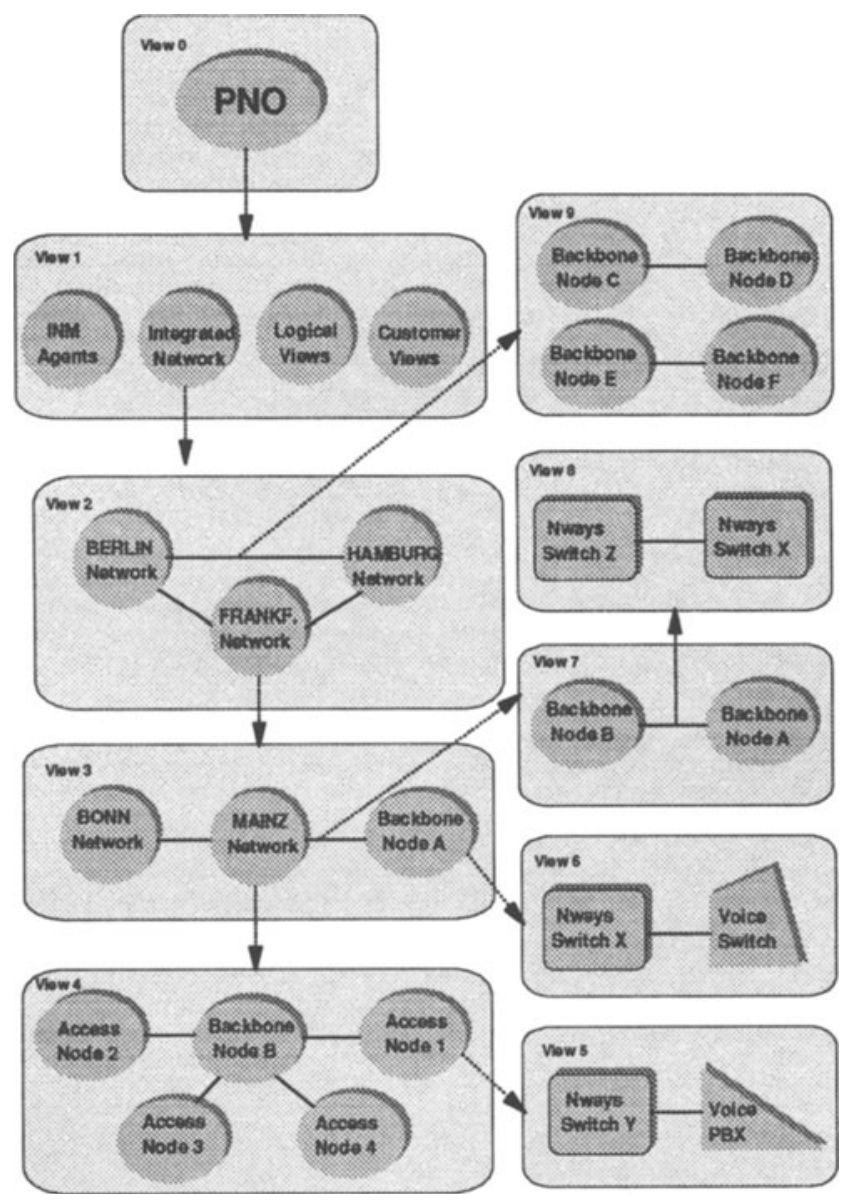

Figure 4 Network View

\section{Exploding Links between Networks and Nodes}

Lines representing links between networks or between networks and nodes can be exploded into sub-maps with the actual links connecting two nodes, each one belonging to one of the termination points of the exploded line, as shown in views 7 and 9 . Note that multiple links may exist and will be presented like in view 9.

\section{Exploding Links between two Nodes}

Lines representing links between two nodes, as shown in view 7 , can be exploded into sub-maps with the actual links connecting two equipment each one belonging to one of the termination points of the exploded line. At least one pair of equipment, connected by a line, will exist and will be presented as in view 8 .

Such lines, will always represent instances of one of the following classes:

- [PN]:pnTrunk

- [PN]:pnVoiceEquipNwaysLink

- [PN]:pnVoiceEquipVoiceEquipLink 


\section{Exploding Nodes of a Network}

Icons representing either a backbone node or an access node can be exploded into a sub-map showing icons representing the actual equipments, like Nways Switches and Voice Equipment. Such sub-maps, with the details of backbone nodes or access nodes as shown in views 5 and 6 , can contain icons representing instances of the following classes, contained in the PNO Integration Agent.

\section{Links between Equipment}

Icons representing Nways Switches and icons representing Voice Equipment can be connected with lines representing that a direct connection exists between them as shown in views 5 and 6 . The lines connecting such icons will always represent instances of one of the following classes:

- [PN]:pnTrunk

- [PN]:pnVoiceEquipNwaysLink

- [PN]:pnVoiceEquipVoiceEquipLink

In the integrated topology of the PNO network only one line will be shown connecting two pieces of equipment even if multiple physical links exist between the two systems. It will also not be possible to explode such line into sub-maps showing details of the physical links that it represents.

\section{Integrated Configuration Management}

Configuration management of the various network elements will bee provided through the Voice Switch Manager (VSM) application and the Nways Switch Manager (NSM) [11], [12], respectively. In the INM an integrated configuration management application for the network level will be provided. The INM configuration management application will support discovery functions for network elements that have been defined in VSM and NSM, and configuration management functions on the level of the OMNIPoint object model. The principle network configuration functions are listed below. The integrated configuration management will provide the functions to change dynamically the configuration of the network:

- add a new network node - allows to introduce a new network node.

- add a new connection - introduces new connections between existing network nodes via an appropriate user interface.

- remove a network node - ensures proper removal of an existing network node (e.g. no links/connections exist for nodes being removed).

- remove a connection - guarantees topology consistency in an analogue way as the respective function for removing network nodes.

- view/change node parameters - allows convenient node parameter changes.

- view/change connection parameters - provided to handle connection parameters.

- add a new location - allows to introduce a location into the network.

- remove a location - removes a defined location from the network.

- define a new subnetwork - allows to structure a big network hierarchically.

- existing subnetwork change - allows to restructure a network hierarchy.

- remove a subnetwork - removes a defined subnetwork leaving a consistent topological view of the higher layer network.

\section{Integrated Fault Management}

Voice switches report alarms to the Voice Switch Manager application, while the Nways switches report alarms to the Nways Switch Manager. The two management systems provide different interfaces and capabilities for handling alarms. In order to provide an integrated process for handling and tracking alarms a unique way for indicating alarms at one centralized point of control is required. Similarly to the integration 
of the different topologies it is intended to integrate the alarm management via a CMIP agent. This allows to utilize the generic Fault Application of TMN/6000 for the integrated centralized fault management. For the VSM the VSM agent will perform the necessary mapping between the VSM message format and the event-report format as described in the standard documents [1], [2], [3].

The integrated Fault Management will provide the following functions:

- display of events/alarms in a textual way (event cards or event lists);

- highlight the network elements in the topology for which an event report has been generated;

- access the TMN browser [10] to retrieve specific information on the network element for which the event report has been generated;

- manage event report workspaces;

- manage Event Forwarding Discriminators (EFDs);

For controlling the event filters in the various agents an operator interface is provided. With this interface new filters can easily be created and maintained.

\section{Customer Network Management}

As part of the INM architecture a customer network management gateway has been introduced to allow a customer to perform an integrated end-to-end management of his private network(s) connected via the PNO network. Our concept for realizing the gateway functionality is based on the X.700 agent/manager framework [3] of the TMN/6000 platform, establishing a service management layer. More precisely, via an agent interface (CMIP/SNMP) customer specific agents are introduced which provide a customer with relevant information extracted from the integrated agent on the network management layer employing manager functions. Realizing a separate agent rather than allowing a customer having direct access to parts of the integrated MIB has the following main advantages:

- enhanced security due to the separated agents;

- scalable performance since customer specific agents can be located on one or several machines.

It has to be mentioned that by providing a CMIP or SNMP agent it is the task of the customer to integrate the agent into his management environment and to look for appropriate applications to manage such an agent. However, since the applications for managing the integrated agent at the network management layer are generic, they can also be exploited for managing the customer agents. Thus, an additional service could be provided to a customer.

The MIB defined in the different customer agents will be derived from the integrated MIB at the network management layer. The chosen information model for the integrated MIB allows to define customer specific subnetworks. Those subnetworks will be extracted and stored in the different customer agents. The manager component of the X.700 agent/manager framework will take care of the synchronization between the customer and the integrated agent.

Functions for administrating the various agents at the service management layer will be provided that allows to:

- add a new customer - introduces a new customer into the PNO network;

- remove a customer - deletes a customer from the PNO network;

- configure a customer network - allows to define the customer specific network components (nodes, links, subnetworks) based on the topology;

- change a customer network - allows to change the configuration associated with a customer via the topology display.

As indicated in the overview of the management system architecture, VPNs are realized as customer specific agents. Those agents are derived from the integration agents and 
cooperate with them. The VPN agents will provide standardized management interfaces upon which standard application can act. To manage VPNs the following functions will be available:

- create a VPN agent - supports the creation of a VPN agent for components of the PNO network. being selected in the topology display;

- list all VPN agents - provides an overview of all created VPN agents;

- view a VPN agent - allows to visualize the configuration of selected VPN agents;

- delete a VPN agent - deletes a generated VPN agent;

\section{SUMMARY}

The architecture, design and realization of a network management system for a large and complex telecommunication network has been presented. The main goal was to provide a well architected solution that fulfills certain requirements with respect to scalability and extendablity and which could still be implemented within a restricted time frame and with a restricted number of resources. With the conceived modular architecture based on standard building blocks and standardized communication interfaces (CMIP) the scalability and the extendability of the whole management system could be assured. The various agents and management applications can be located on different computers or colocated on the same machine. Depending on the size of the network the appropriate hardware configuration for the management system can be chosen. New management applications can be integrated utilizing standardized interfaces. New network elements can be covered extending the object model appropriately.

\section{REFERENCES}

[1] ITU-T Recommendations M.3000 - Overview of TMN Recommendation - 1994

[2] ITU-T Recommendations M.3010 - Priciples for a Telecommunications Management Network - 1994

[3] CCITT Recommendation X.700, Management Framework Definition for Open Systems Interconnection (OSI) for CCITT Applications

[4] CCITT Recommendations X.722 (1992) / ISO/IEC 10165-4: 1992, „Guidelines for the Definition of Managed Objects"

[5] NMF: Forum 006, Forum Library - Vol.4: OMNIPoint 1 Definitions, 1992

[6] NMF: Forum 019, OMNIPoint 1 Object and Ensemble List, 1992

[7] Veli Salin, „Telecommunications Management Network, Principles, Models, and Applications", Telecommunications Network Management into the 21 st Century, 1993, p.72-135

[8] GC31-8016, IBM TMN Products for AIX: General Information, 1995

[9] GC31-8008, IBM TMN Products for AIX: Developing TMN Applications, 1995

[10] SC31-8017, IBM NetView TMN Support Facility for AIX: User's Guide, 1995

[11] SH11-3072, IBM Nways BroadBand Switch Manager for AIX, Getting Started, 1995

[12] SH11-3073, IBM Nways BroadBand Switch, Distributing and Updating the Nways Switch Manager and Nways Switch Manager Control Program, 1995 